\title{
Increased elastin turnover in diabetic patients with arterial hypertension
}

\author{
ASPARUH NIKOLOVI, IVAN TSINLIKOVI, GEORGE NICOLOFF², ALEXANDER BLAZHEV', \\ IVANKA TSINLIKOVA ${ }^{l}$, SPASKA STANILOVA ${ }^{3}$, ANTOAN GAREV $V^{l}$ \\ ${ }^{1}$ Department of Propedeutics of Internal Diseases, Medical University, Pleven, Bulgaria \\ ${ }^{2}$ Division of Biology and Immunology, Medical University, Pleven, Bulgaria \\ ${ }^{3}$ Department of Biology, Trakia University, Stara Zagora, Bulgaria
}

\begin{abstract}
Introduction and aims: Antibodies against products of elastin degradation are found in sera of all people. Presence of anti-elastin antibodies (AEAbs) and relevant antigens in circulation leads to the formation of circulating immune complexes (CIC). The aim of our study is to determine serum levels of anti-elastin antibodies unbound in CIC (free AEAbs).

Material and methods: We used a method for detection CIF-ELISA (complement-inhibiting factor-enzyme linked immunosorbent assay) in combination with ELISA to find AEAbs. The levels of free AEAbs IgG were measured in sera of 93 patients with diabetes mellitus and hypertension (mean age $61.4 \pm 11.3$, diabetes duration $9.88 \pm 3.12$; hypertension duration $9.28 \pm 4.98$ ). These levels were compared to 42 age- and sex-matched controls.

Results: Free AEAbs IgG in patients with T2DM and AH are statistically significantly higher than these in healthy controls: 0.421 (0.328-0.572) vs. 0.240 (0.212-0.305), respectively $(K W=19.64 ; p<0.0001)$. Group 1 (patients with microvascular complications) showed a significant increase in free AEAbs IgG in comparison with controls: $0.428(0.343-0.591)$ vs. $0.240(0.212-0.305)$, respectively $(K W=20.14$; $p<0.0001)$. Group 2 also shows higher levels of free AEAbs IgG than the control group: 0.398 (0.312$0.467)$ vs. $0.240(0.212-0.305)$, respectively $(K W=8.88$; $p=0.003)$. Patients with microvascular complications showed the highest levels of free AEAbs IgG. There were no significant differences between group 1 and group 2.

Conclusions: Our results show the association between the activity of elastin turnover and microvascular lesions in diabetic patients with arterial hypertension. We suggest that free AEAbs IgG mark a later "secondary" step of the autoimmunization to elastin.
\end{abstract}

Key words: ELISA, diabetes mellitus, elastin, arterial hypertension, microangiopathy.

(Centr Eur J Immunol 2013; 38(4): 537-542)

\section{Introduction}

Patients with type 2 diabetes mellitus are at a high risk of developing diabetic microvascular complications due to the impaired structure of the vascular protein elastin and collagen type IV. The elastic fibers, responsible for the compliance and elasticity, are organized in elastic lamellae. These structures separate layers of smooth muscle cells and equally distribute the pressure along the entire vascular wall. The degradation or calcification of elastic fibers in many diseases, affecting the small caliber vessels, leads to vascular damages. Our previous studies in patients with type 1 diabetes mellitus found an increased degradation of elastin - the major protein of elastic fibers. As a result, soluble elastin-degradation peptides (EDP) are released in the circulation and act as a pathological stimulus for the formation of anti-elastin antibodies (AEAbs) [2]. In type 2 diabetes mellitus, different types of autoantibodies have been detected: insulin autoantibodies, GAD-antibodies (glutamic acid decarboxylase antibodies), autoantibodies to tyrosine phosphatase IA2, islet antibodies [3], anti-elastin antibodies [2] and collagen type IV autoantibodies. These autoimmune antibodies bind to cognate antigens and thus, form circulating immune complexes (CIC). These CIC have pathogenic properties because they are deposited in the small caliber vessels, accelerating their damage.

\section{Material and methods}

In our previous studies, we used a method based on C3-binding glycoprotein: complement-inhibiting factor CIF-enzyme linked immunosorbent assay (CIF-ELISA) 
(developed by Stanilova and Slavov) [5]. The method was used to determine CIC (IgG, IgM, and IgA) in the sera of diabetic patients [6]. We established a correlation between CIC IgG class and the development of vascular damages. In the present study, we used CIF-ELISA combined with ELISA in order to determine AEAbs with the following steps: a) elimination of AEAbs incorporated in $\mathrm{CIC}$; b) measurement of free serum anti-elastin $\mathrm{IgG}$, unbound in CIC; c) testing the possible correlation between free AEAbs IgG and the development of microvascular damages in patients with diabetes and arterial hypertension.

The sera of 42 clinically healthy subjects (mean age $58.9 \pm 7.56)$ were used as controls. The selected controls were individuals with no family history of arterial hypertension, diabetes mellitus and atherosclerosis, who did not suffer from any inflammatory processes, collagenoses, and emphysema and had no history of hepatitis. Their routine clinical examinations, lipid profile and serum proteins showed no changes, ECG was normal.

Apart from the clinically healthy individuals, 93 patients with type 2 diabetes mellitus and arterial hyperten- sion were also examined. Their mean age was $61.4 \pm 11.3$ years; the duration of diabetes was $9.88 \pm 3.12$ years, and of arterial hypertension $-9.28 \pm 4.98$ years. They were all from the region of the Medical University in Pleven. The diabetic patients were divided into two groups according to the presence - group $1(n=67)$ or absence - group 2 $(n=26)$ of microangiopathy (Tables 1 and 2). An ethical approval was obtained from the Ethics Committee, and the patients signed informed consent forms for their participation in the research.

Ophthalmoscopy through dilated pupils was carried out in all patients with diabetes mellitus to assess the presence of retinopathy; all patients were examined by the same ophthalmologist.

Glycated hemoglobin A1c was measured using highpressure liquid chromatography (normal range 4-6\%).

For the measurement of plasma glucose, the blood was collected in test tubes with glycolytic inhibitor and analyzed in the central laboratory within 3-4 h, using the GOD-PAP method (Boehringer Mannheim, Mannheim, Germany) with Hitachi 705 autoanalyzer (Boehringer Mannheim).

Table 1. Clinical data of patients with type 2 diabetes mellitus and arterial hypertension

\begin{tabular}{|c|c|c|c|}
\hline Clinical data & Group 1 & Group 2 & Controls \\
\hline age & $62.5 \pm 12.58$ & $60.4 \pm 8.4$ & $58.9 \pm 7.56$ \\
\hline sex (male/female) & $26 / 41$ & $11 / 15$ & $20 / 22$ \\
\hline average duration of diabetes & $9.30 \pm 5.36$ & $9.16 \pm 7.59$ & N/A \\
\hline $\begin{array}{l}\text { average duration } \\
\text { of hypertension }\end{array}$ & $9.50 \pm 7.63$ & $8.68 \pm 7.26$ & N/A \\
\hline $\mathrm{HbA}_{1 \mathrm{c}}$ & $7.63 \pm 2.03 *$ & $7.27 \pm 1.63$ & N/A \\
\hline systolic AH (mm Hg) & $142.83 \pm 18.05$ & $140.58 \pm 20.51$ & $114.29 \pm 15.74$ \\
\hline diastolic $\mathrm{AH}(\mathrm{mm} \mathrm{Hg})$ & $82.23 \pm 11.52$ & $81.35 \pm 11.96$ & $72.5 \pm 10.4$ \\
\hline BMI & $29.62 \pm 4.99$ & $28.42 \pm 3.96$ & $22.61 \pm 2.27$ \\
\hline common cholesterol (mmol/l) & $5.26 \pm 1.40 *$ & $5.18 \pm 0.93$ & $3.99 \pm 0.65$ \\
\hline $\mathrm{HDL}(\mathrm{mmol} / \mathrm{l})$ & $0.88 \pm 0.30^{*}$ & $0.93 \pm 0.30$ & $0.96 \pm 0.20$ \\
\hline $\operatorname{LDL}(\mathrm{mmol} / \mathrm{l})$ & $3.18 \pm 1.19$ & $3.16 \pm 1.09$ & $2.43 \pm 0.64$ \\
\hline triglycerides (mmol/l) & $2.91 \pm 1.68$ & $2.53 \pm 1.49$ & $1.31 \pm 0.61$ \\
\hline insulin dose (U/kg/24 h) & $2.57 \pm 0.52$ & $2.03 \pm 0.93$ & N/A \\
\hline $\mathrm{MAU}(\mu \mathrm{g} / \mathrm{min})$ & $78.94 \pm 52.87 *$ & $8.53 \pm 4.69$ & $\mathrm{~N} / \mathrm{A}$ \\
\hline MAU & $(n=43)$ & - & \\
\hline retinopathy & $(n=20)$ & - & \\
\hline neuropathy & $(n=4)$ & - & \\
\hline smokers & $37 / 67$ & $15 / 26$ & $16 / 42$ \\
\hline number of patients & 67 & 26 & 42 \\
\hline
\end{tabular}

Group 1 - patients with vascular complications $(n=67) ;$ group 2 - patients without vascular complications $(n=26) ;$ controls $(n=42)$. Values are mean $\pm S D$ 
Total serum cholesterol and triglyceride concentrations were measured by enzyme assay (Boehringer Mannheim, Mannheim, Germany).

Arterial blood pressure was measured using a standard mercury sphygmomanometer, to the nearest $2 \mathrm{~mm} \mathrm{Hg}$, in the dominant arm after at least $10 \mathrm{~min}$ rest in the supine position.

Albumin excretion rate (AER) was determined by nephelometry with a commercial kit containing specific antibody (Behringwerke, Marburg, Germany).

\section{Antigen}

The antigen soluble $\alpha$-elastin was prepared from the aorta of healthy individuals (who died in an accident) in a way already described (Nicoloff et al., 2000) [7]. Elastin purity was confirmed by the amino acid analysis of Professor R. Mecham (Washington University, St Louis, MO). Circulating AEAbs were determined using a twostep method: CIF-ELISA for elimination of the immune complexes, followed by elastin-specific ELISA for determination of AEAbs.

CIF-ELISA plates were prepared by incubation of the wells with CIF $(20 \mu \mathrm{g} / \mathrm{ml}$ in $0.2 \mathrm{M}$ carbonate-bicarbonate buffer, pH 9.6 overnight at $4^{\circ} \mathrm{C}$ ). CIF was isolated from the seeds of a parasitic plant Cuscuta europaea. After the plates had been washed repeatedly in order to remove unbound CIF, human sera $(100 \mu \mathrm{l})$ were added to the plates and incubated for $60 \mathrm{~min}$ at $37^{\circ} \mathrm{C}$. At the end of the incubation period, the samples were transferred from the CIF plates to the wells, sensitized with aortic $\alpha$-elastin $(1 \mu \mathrm{g}$ of elastin in $100 \mu$ l of $0.05 \mathrm{M}$ carbonate buffer, $\mathrm{pH}$ 9.6) in a previously described manner [7]. These samples were then incubated for $1 \mathrm{~h}$ at $37^{\circ} \mathrm{C}$ and washed several times. The conjugated antibody was found using goat anti-human immunoglobulin peroxidase conjugates (SIGMA, USA), diluted $1: 10,000$ with PBS, containing $1 \%$ bovine serum albumin (BSA) and $0.05 \%$ Tween 20 as well as o-Phenylenediamine $(4 \mathrm{mg}$ in $10 \mathrm{ml}$ of $0.05 \mathrm{M}$ citrate buffer, $\mathrm{pH}$ 5.0) and $0.01 \% \mathrm{H}_{2} \mathrm{O}_{2}$. The plate was incubated for $1 \mathrm{~h}$ at room temperature in a dark chamber. The reaction was stopped by adding $4 \mathrm{M}$ of $\mathrm{H}_{2} \mathrm{SO}_{4}$ to each well. The optical density was measured with a Microelisa Reader 210 (Organon Teknika, Belgium) at a wavelength of $492 \mathrm{~nm}$.

\section{Controls}

1. Substrate control: the substrate solution was added directly to the plates with elastin.

2. Immunoconjugate control: $\operatorname{IgG}$ immunoconjugate was tested in polystyrene wells, sensitized with BSA and in wells, coated with other human immunoglobulins (IgM, $\operatorname{IgA}$ and $\operatorname{IgD})$.

3. Inhibition of ELISA: 10 serum samples with the highest levels of AEAbs were pre-incubated with $100 \mu \mathrm{g}$ of hu-
Table 2. Distribution (in percents) of patients in the groups according to sex and presence or absence of smoking as a factor

\begin{tabular}{lcccc}
\hline & \multicolumn{2}{c}{ Sex } & \multicolumn{2}{c}{ Smoking } \\
\cline { 2 - 5 } & male & female & smokers & $\begin{array}{c}\text { non- } \\
\text { smokers }\end{array}$ \\
\hline Group 1 & $39 \%$ & $61 \%$ & $55 \%$ & $45 \%$ \\
\hline Group 2 & $42 \%$ & $58 \%$ & $58 \%$ & $42 \%$ \\
\hline Controls & $45 \%$ & $55 \%$ & $47 \%$ & $53 \%$ \\
\hline
\end{tabular}

man $\alpha$-elastin for $30 \mathrm{~min}$ at $37^{\circ} \mathrm{C}$; the mixture was then centrifuged for $30 \mathrm{~min}$ at $15000 \mathrm{~g}$. Supernatants for ELISA were used following the usual protocols.

4. CIF-ELISA control: after the absorption of IgG CIC by CIF, $100 \mu \mathrm{l}$ of absorbed serum was tested for the presence of IgG CIC.

All samples were tested three times. The peripheral wells were not used in order to avoid edge effects.

\section{Statistical analysis}

The research data were processed with the computer programs EXCEL (Microsoft Corporation, Redmond, WA) and STATGRAPHICS plus (Manugistics, Rockville, MD) for WINDOWS. All results were described in tables, graphs, numerical values (mean $\pm \mathrm{SD}$, share indicators and correlations). For assessment and conclusions in the case of normal distribution, the Student $t$-test, Fisher's F-test (ANOVA) and post-hoc tests (LSD, Tukey HSD, Scheffe, Bonferroni, Newman-Keuls, and Duncan) were used, and for distribution, different from the normal - the K-W (Kruskal-Wallis)-test. The level of significance was determined as $p<0.05$.

\section{Results}

Free AEAbs IgG in patients with T2DM and AH are statistically significantly higher than these in healthy controls: $0.421(0.328 \div 0.572)$ vs. $0.240(0.212 \div 0.305)$, respectively $(\mathrm{KW}=19.64 ; p<0.0001)$. Group 1 (patients with microvascular complications) showed a significant increase in free AEAbs IgG in comparison with controls: $0.428(0.343 \div 0.591)$ vs. $0.240(0.212 \div 0.305)$, respectively $(\mathrm{KW}=20.14 ; p<0.0001)$. Group 2 also shows higher levels of free AEAbs IgG than the control group: 0.398 $(0.312 \div 0.467)$ vs. $0.240(0.212 \div 0.305)$, respectively $(\mathrm{KW}=8.88 ; p=0.003)$. Patients with microvascular complications showed the highest levels of free AEAbs IgG. There were no significant differences between Group 1 and Group 2. Free AEAbs showed a correlation with HbA1c $(r=0.22) ;(p=0.04)$, duration of diabetes $(r=0.18)$; $(p=0.05)$, systolic blood pressure $(r=0.15) ;(p=0.05)$, 
Table 3. Values of free serum anti-elastin antibodies (free AEAbs) in patients with type 2 diabetes mellitus and arterial hypertension

\begin{tabular}{lcccc}
\hline \multirow{2}{*}{ Groups } & free AEAbs $(\mathbf{n g} / \mathbf{m l})$ & \multicolumn{3}{c}{ Comparison to other groups } \\
\cline { 2 - 5 } & Mean \pm SD & Group 1 & Group 2 & All patients \\
\hline all patients & $0.421(0.328 \div 0.572)$ & NS & NS & NS \\
\hline group 1 & $0.428(0.343 \div 0.591)$ & - & NS & NS \\
\hline group 2 & $0.398(0.312 \div 0.467)$ & NS & $p=0.003$ & $p<0.0001$ \\
\hline controls & $0.240(0.212 \div 0.305)$ & $p<0.0001$ & $p$
\end{tabular}

Free AEAbs in patients with T2DM and AH are statistically significantly higher as compared to controls $(p<0.0001)$. Group 1 showed a significant elevation in the levels of free AEAbs as compared to healthy individuals $(p<0.0001)$. Group 2 also showed an increase in serum free AEAbs in comparison to the controls

$$
(p=0.003)
$$

total cholesterol $(r=0.20) ;(p=0.03)$, and triglycerides $(r=0.21) ;(p=0.02)$.

\section{Discussion}

Arterial hypertension and diabetic vascular complications are connected with an elevated degradation of elastic tissue. As a result, soluble elastin peptides are released to the circulating blood and act as a pathologic stimulus for an increased production of anti-elastin antibodies. Because it is very important to find characteristics of pathological activation of elastin turnover, we studied diabetic patients with arterial hypertension. Our data suggest an association between the activity of elastin turnover (especially increased serum levels of free IgG AEAbs) and vascular complications. Elastin is one of the main proteins in the vascular wall and elastin degradation is accelerated in diabetic patients. The formation of EDP, free AEAbs IgG and elastin-antielastin immune complexes may play an essential role in progression of the late complications of diabetes mellitus.

Humoral and cell-mediated mechanisms take part in the start and progression of atherogenesis. The role of autoantibodies and immune complexes in this process attracts an increasing attention of researchers. The antigenic property of elastin and the circulating AEAbs were first reported by Stein et al. [9] and confirmed by several subsequent studies [7, 10-13]. At present, two main antigen classes are recognized in the elastin molecule: one is specific, the other one - non-specific and associated with the cross reactivity. Normally AEAbs IgG as well as IgA, IgM, IgD can be found in very low levels in the human body [14]. AEAbs from different immunoglobulin classes are present also in pathological conditions, but at much higher levels [2, 14-18]. $\alpha$-elastin is an oxaloacetic soluble product of the insoluble "native" elastin. The oxalic acid separates the elastin chain in an unspecific manner, creating a heterogeneous population of peptides varying from 100 to 10 $\mathrm{kDa}$. Thus, a great number of antigenic sites are formed for maximum reactivity.
In our previous study in diabetic patients, we found that the increase in AEAbs IgM and IgG suggests an initial pathological autoimmunization to human elastin while the elevation of AEAbs IgA levels - a late accelerated elastin degradation and development of vascular disease [2]. In these studies, however, it was not possible to specify which of AEAbs are components of immune complexes and which are free in the circulation. The reason is that not all of AEAbs paratopes are bound to the relevant elastin epitopes. This is why some of the free paratopes in AEAbs, incorporated in elastin-antielastin CIC react with human aorta $\alpha$-elastin during standard ELISA for AEAbs detection. The immunoconjugates, used to determine AEAbs, are specific to the immunoglobulin heavy chain, so it is not possible to assess if AEAbs found are incorporated in CIC.

In our earlier research, we revealed the presence of CIC (IgG, IgM and IgA) in the sera of diabetic patients using the CIF-ELISA method [6]. In the present study, we used the ability of CIF-ELISA to detect and absorb CIC, containing various immunoglobulin isotypes, and thus to remove all CIC from the serum samples. As a next step, ELISA was used to measure the levels of AEAbs, unbound in immune complexes.

Our results show that the values of free AEAbs IgG in patients with vascular complications are significantly higher than in patients without vascular damages and healthy controls. This means that the diabetics from Group 1 show pathologically high immune response to elastin.

Although the data from Group 2 reveals levels of free AEAbs higher than those of the controls, these levels still remain lower than those measured in patients with vascular complications from Group 1. It is extremely important to monitor if the group of patients with high levels of free AEAbs will develop vascular lesions before the patients without such elevated levels of free AEAbs.

The information that elastin has antigen properties leads to the hypothesis that EDP, found in vivo as a result of degradation or synthesis, can induce an autoimmune response [18-20]. It is possible that some of free AEAbs that we detected form immune complexes with corresponding EDP. The elastin can become a substrate for the formation 
of insoluble immune complexes (at tissue level) or soluble CIC in the serum. Group 1 patients probably represent a "secondary" active phase in the development of vascular damages due to increased synthesis of free AEAbs IgG. There was a correlation established between free AEAbs on the one hand and $\mathrm{HbA}_{1 \mathrm{c}}(r=0.22) ;(p=0.04)$, total cholesterol $(r=0.33)$; $(p=0.05)$, triglycerides $(r=0.38)$; $(p=0.03)$, microalbuminuria $(r=0.41) ;(p=0.002)$, on the other hand.

A possible explanation of this result is the evidence that the first immunoglobulin synthesized during the early phase of a pathologically activated immune response is IgM. The immune system then switches on to production of IgG. The elevation of AEAbs of the IgG types is therefore the second indicator of the pathological turnover of elastin and the development of vascular complications in diabetics.

In our study it was not possible to detect the "active" phase of vascular disease, because of patients' diabetes duration of $9.88 \pm 3.12$ years. During this "active" phase, the levels of IgM were probably elevated, while during the chronic phase levels of free AEAbs IgG were increased. The presence of free AEAbs IgG could lead to a variety of pathological processes including immune complexes formation, complement activation and $\mathrm{K}$ cell-mediated antibody-dependent cell-mediated cytotoxicity (ADCC) activation, all of which may contribute to the progression of elastin destruction of the arterial wall. Furthermore, elastin antigen present at the damaged arterial sites retains selectively antigen specific T- (Peterszegi et al., 1997) [30, 31] and B-lymphocytes which would aggravate the response.

The elastic fibers may be involved in the process of lipid precipitation in arteries [21]. Our results support these findings. Free AEAbs showed a correlation with the triglycerides and total cholesterol. The importance of the lipid profile (increased triglyceride and total cholesterol values) should also be emphasized [22-24]. The elevated levels of serum triglycerides which contain multiple types of potential atherogenic lipoproteins enrich the picture of diabetic dyslipidemia [25]. It is the most common deviation in poorly controlled diabetes. In patients with microvascular complications, the attachment of triglycerides to the arterial wall can lead to the conversion of elastin into an immunogenic form. In the present study, the triglycerides were elevated over the normal range in patients with vascular complications, which supports the idea about this feature.

We interpreted the correlation between free AEAbs and $\mathrm{HbA}_{1 \mathrm{c}}$ in Group 1. It is known that diabetes duration and age correlate with the development of vascular complications. Glycation of hemoglobin leads to the formation of $\mathrm{HbA}_{1 \mathrm{c}}$, which is described as a product of Amadori, but it is not a product of the late non-enzymatic glycosylation [26]. $\mathrm{HbA}_{1 \mathrm{c}}$ is an indicator for glycemia over the last 6-12 weeks while the late non-enzymatic glycosylation marks a process longer in time [27].

Bako et al. [28] in order to investigate the role of the immune system in the arteriosclerotic process, we investigated the anti-elastin peptide antibodies (AEAb) of the IgG and IgM types by DOT immunobinding assay in the sera of patients suffering from various arteriosclerotic diseases. In total 232 control and pathological sera were studied. In obliterative arteriosclerosis of the legs $-90 \%$, ischemic heart disease $-67 \%$ and hypertension $-60 \%$ of sera were positive for AEAb of the IgG type independent of age. In the case of diabetes mellitus, however, the duration of the disease was a determinant. In rheumatoid arthritis, the results were negative. No clear-cut positivity could be demonstrated in stroke patients, either. These results indicate that AEAb can be detected in some diseases and DOT appears to be an appropriate method for the AEAb screening in various diseases.

Gmiński et al. [30] further investigate anti-elastin antibodies of the IgG and IgM types in sera of patients suffering from lung cancer, using the DOT immunobinding assay. Authors studied 96 pathological and 40 control sera. Anti-elastin antibodies were found to be present in $45 \%$ of patients with small cell lung cancer, $19 \%$ of subjects with adenocarcinoma and not-identified lung tumor and $15 \%$ of patients with squamous cell lung cancer. They circulated in $5 \%$ of control persons only. The highest values of their titers were observed in the advanced stages of disease. In $55 \%$ of anti-elastin antibody positive small cell lung cancer patients, antibodies were of the IgM type, suggesting the initial step of the autoimmunization to elastin.

AEAbs and EDP are markers for elastin degradation. The production of AEAbs by the immune system is a dynamic process in which some of these antibodies are consumed by binding to EDP while the rest remain free in the circulation. We observed the highest levels of free AEAbs in patients with vascular complication and this is why we formed the hypothesis that free AEAbs IgG mark a later "secondary" step of the autoimmunization to elastin.

In conclusion, our results suggest an association between the activity of elastin turnover and microvascular lesions in diabetic patients with arterial hypertension. Elevation of serum free AEAbs IgG levels may indicate increased elastin degradation and development of microvascular complications. However, a larger study is necessary for clarification of these possibilities.

The authors declare no conflict of interests. 


\section{References}

1. Nicoloff G, Baydanoff S, Stanimorova N, et al. (2000): Relationship between elastin-derived peptides and the development of diabetic microvascular complications - a longitudinal study in children with type 1 (insulin-dependent) diabetes mellitus. Gen Pharmacol 35: 59-64.

2. Nicoloff G, Baydanoff S, Stanimirova N, et al. (2000): An association of anti-elastin IgA antibodies with development of retinopathy in diabetic children. Gen Pharmacol 35: 83-87.

3. Verge CF, Stenger D, Bonifacio E, et al. (1998): Combined use of autoantibodies IA-2 autoantibody, GAD autoantibodies, insulin autoantibodies, cytoplasmic islet cell autoantibodies in type 1 diabetes: combinatorial islet autoantibody workshop. Diabetes 47: 1857-1866.

4. Nicoloff G, Baydanoff S, Petrova Ch, Christova P (2002): Serum antibodies to collagen type IV and development of diabetic vascular complications in children with type 1 (insulin-dependent) diabetes mellitus - a longitudinal study. Vascul Pharmacol 38: 143-147.

5. Stanilova SA, Slavov ES (2001): Comparative study of circulating immune complexes quantity detection by three assays - CIF-ELISA, C1q-ELISA and anti-C3 ELISA. J Immunol Methods 2001; 253: 13-21.

6. Nicoloff G, Blazhev A, Petrova C, Christova P (2004): Circulating immune complexes among diabetic children. Clin Dev Immunol 11: 61-66.

7. Baydanoff S, Nicoloff G, Alexiev C (1987): Age-related changes in anti-elastin antibodies in serum from normal and atherosclerotic human subjects. Atherosclerosis 63: 267-271.

8. Zhelev ZD, Stanilova SA, Carpenter BG (1994): Isolation, partial characterization and complement inhibiting activity of a new glycoprotein from Cuscuta europea. Biochem Biophys Res Commun 202: 186-194.

9. Stein F, Pezess MP, Robert L, Poullain N (1965): Anti-elastin antibodies in normal and pathological human sera. Nature 207: 312-313.

10. Daynes RA, Thomas M, Alvarez VL, Sandberg LB (1977): The antigenicity of soluble porcine elastins: I. Measurement of antibody by a radioimmunoassay. Connect Tissue Res 5: 75-82.

11. Mecham RP, Lange G (1982): Antigenicity of elastin: characterization of the major antigenic determinants on purified insoluble elastin. Biochemistry 21: 669-673.

12. Wrenn DS, Mecham RP (1987): Immunology of elastin. Methods Enzymol 144: 246-259.

13. Baydanoff S, Nicoloff G, Russev T, Alexiev C (1988): Detection of elastin-antielastin circulating immune complexes (CIC) in diabetic patients with vascular damage. Cor Vasa 30: 361-367.

14. Baydanoff S, Nicoloff G, Alexiev C (1991): Age-dependent changes in the level of antielastin antibodies of different immunoglobulin classes (IgG, IgM, IgA and $\operatorname{IgD}$ ) in human serum. Cor Vasa 33: 197-205.

15. Fulop T, Wei S, Robert L, Jacob MP (1990): Determination of elastin peptides in normal and atherosclerotic human sera by ELISA. Clin Physiol Biochem 8: 273-278.

16. Gmiński J, Drózdz M, Ulfig-Maślanka R, Najda J (1991): Evaluation of elastin metabolism in children from families with high risk of atherosclerosis. Atherosclerosis 91: 185-189.

17. Daskalova M, Taskov H, Dimitrova E, Baydanoff S (1997): Humoral and cellular immune response to elastin in patients with systemic sclerosis. Autoimmunity 25: 233-241.
18. Colburn KK, Langga-Shariffi E, Kelly GT, et al. (2003): Abnormalities of serum antielastin antibodies in connective tissue diseases. J Investig Med 51: 104-109.

19. Péterszegi G, Mandet C, Texier S, et al. (1997): Lymphocytes in human atherosclerotic plaque exhibit the elastin-laminin receptor and potential role in atherogenesis. Atherosclerosis 135: 103-107.

20. Péterszegi G, Texier S, Robert L (1997): Human helper and memory lymphocytes exhibit an inducible elastin-laminin receptor. Int Arch Immunol 114: 218-223.

21. Podet EJ, Shaffer DR, Gianturco SH, et al. (1991): Interaction of low density lipoproteins with human aortic elastin. Atheroscler Thromb 11: 116-122.

22. Microalbuminuria Collaborative Study, Group; United Kingdom (1993): Risk factors for development of microalbuminuria in insulin dependent diabetic patients: a cohort study: Microalbuminuria Collaborative Study Group, U.K. BMJ 306: 1235-1239.

23. Poulsen PL, Hansen KW, Mogensen CE (1994): Ambulatory blood pressure in the transition from normo- to microalbuminuria. A longitudinal study in IDDM patients. Diabetes 43: 1248-1253.

24. Mortensen HB, Hougaard P (1997): Comparison of metabolic control in a cross-sectional study of 2,873 children and adolescents with IDDM from 18 countries. Diabetes Care 20: 714-720.

25. Taskinen MR (2003): Diabetic dyslipidaemia: from basic research to clinical practice. Diabetologia 46: 733-749.

26. Kennedy L (2003): Glycation of immunoglobulins and serum proteins. Diabetologia 46: 733-749.

27. Singh R, Barden A, Mori T, Beilin L (2001): Advanced glycation end-products: a review. Diabetologia 44: 129-146.

28. Bako G, Jacob MP, Fulop T Jr, et al. (1987): Immunology of elastin: study of anti-elastin peptide antibodies by DOT immunobinding assay. Immunol Lett 15: 187-192.

29. Fulop T, Wei S, Robert L, Jacob MP (1990): Determination of elastin peptides in normal and atherosclerotic human sera by ELISA. Clin Physiol Biochem 8: 273-278.

30. Gmiński J, Mykała-Cieśla J, Machalski M, Drózdz M (1992): Anti-elastin antibodies in patients with lung cancer. Immunol Lett 33: 211-215.

31. Péterszegi G, Mandet C, Texier S, et al. (1997): Lymphocytes in human atherosclerotic plaque exhibit the elastin-laminin receptor: potential role in atherogenesis. Atherosclerosis 135: 103-107.

32. Péterszegi G, Texier S, Robert L (1997): Human helper and memory lymphocytes exhibit an inducible elastin-laminin receptor. Intl Arch Allergy Immunol 114: 218-223. 